\title{
Solitary choroidal metastasis from gastric adenocarcinoma: a case report
}

\author{
Masanari Shimada ${ }^{1 *}$ D, Susumu Amaya ${ }^{1}$, Hiroaki Sugita ${ }^{1}$, Yuichiro Furutani ${ }^{1}$, Yoshinori Munemoto ${ }^{1}$, Takeshi Mitsui ${ }^{1}$, \\ Toshiro Tanahashi ${ }^{2}$ and Yuzo Kikuchi ${ }^{3}$
}

\begin{abstract}
Background: Metastatic choroidal carcinomas that originated from the gastrointestinal tract are extremely rare. We report a case of suspected solitary choroidal metastasis from gastric adenocarcinoma.

Case presentation: The patient was a 60-year-old man who had undergone laparoscopic distal gastrectomy with D1+ lymphadenecetomy for gastric cancer. The clinical stage was T1bNOMO (TNM classification), but the pathological stage was T4aNOMO beyond expectation. Adjuvant chemotherapy with oral Tegafur, Gimeracil, Oteracil potassium (TS-1 ${ }^{\circ}$ ) was initiated. But he suddenly complained of decreased visual acuity in his right eye about 8 months later. This was suspected to be caused by choroidal metastasis of gastric adenocarcinoma. Chemotherapy with paclitaxel (PTX) and intensity-modulated radiotherapy (IMRT) achieved complete remission and spared the patient from going blind.
\end{abstract}

Conclusions: This case demonstrates that we should be aware of the possibility of choroidal metastases, when visual symptoms arise during treatment of gastric cancer.

Keywords: Gastric cancer, Adenocarcinoma, Choroidal metastasis, Adjuvant chemotherapy, Solitary

\section{Background}

Choroidal metastatic tumors from gastrointestinal tract malignancies are extremely rare compared with more common metastatic breast and pulmonary carcinomas. The uvea consists of the iris, ciliary body, and choroid. In a study of 420 patients with uveal metastasis, 196 came from primary cancers of the breast (47\%), 90 from the lung (21\%), and 18 from the gastrointestinal tract (4\%). Moreover, metastasis was found in the iris in 90 (9\%), ciliary body in $22(2 \%)$, and choroid in $838(88 \%)$ of the 950 metastatic foci in the uvea [1]. Above all, choroidal metastases from gastric adenocarcinoma have been reported in only a few cases in the literature [2-4]. We report a rare case diagnosed as solitary right choroidal metastasis from gastric adenocarcinoma that was treated successfully.

\section{Case presentation}

A 60-year-old man was detected with an abnormality in his medical checkup. Esophagogastroduodenoscopy

\footnotetext{
*Correspondence: masanari.shimada@gmail.com

'Department of Surgery, Fukui-ken Saiseikai Hospital, 7-1

Wadanaka-Funabashi, Fukui 918-8503, Japan

Full list of author information is available at the end of the article
}

revealed an ulcerative lesion on the lesser curvature of the stomach (Fig. 1a). Gastric biopsy specimens from the ulcerative lesion showed signet ring cell carcinoma. We performed computed tomography (CT) and fluorodeoxyglucose-positron emission tomography (FDG-PET), but this did not reveal the primary lesion or lymphnode metastasis (Fig. 1b). We made the clinical diagnosis of T1bNOMO gastric cancer in TNM classification and performed a laparoscopic distal gastrectomy with D1+ lymphadenectomy (Fig. 1c, d). Pathological findings showed exposure of the tumor at the serosal surface at only a single site in the specimen (Fig. 2a, b). We could not recognize the tumor penetration of the serosa intraoperatively. The finding of (tumor exposes on the surface of serosa) T4a was first detected microscopically. The final pathological diagnosis was MU, less, post, type 0-IIc+III (advanced), $30 \times 23 \mathrm{~mm}$, por $2>$ sig, pT4a, sci, INFc, ly1, v1, pN0 (0/40), PM0 (8 mm), and DM0 (90 mm, HER2 score 0 ). We performed preoperative endoscopic marking by clips to $2-\mathrm{cm}$ endoscopic margin and confirmed that the clips were existed in the resected specimen. But, the proximal resected margin was $8 \mathrm{~mm}$ in pathological findings, because the submucosal invasion 


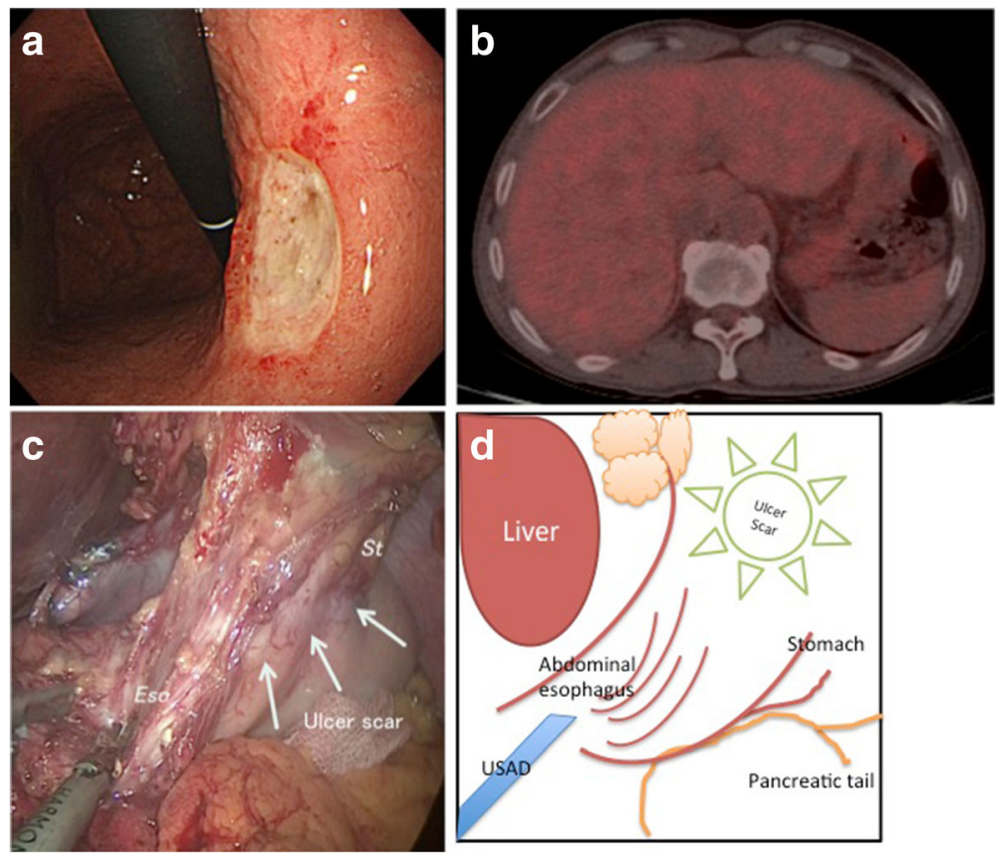

Fig. 1 Preoperative examinations and intraoperative findings. a Esophagogastroduodenoscopy revealed an IIc+III lesion and a signet ring cell carcinoma on biopsy. $\mathbf{b}$ FDG-PET/CT could not detect other lymphnode or organ metastases and could not detect the exposure of the tumor at the serosal surface of the stomach. c The lesion was identified as an ulcer scar during laparoscopic distal gastrectomy. St: Stomach, Eso: esophagus. d Schema of Fig. 1C. USAD: ultrasonic-activated device

was more widespread than the macroscopic margin. Thus, the pathological stage was T4aNOM0 beyond expectation. He began a year of adjuvant chemotherapy with Tegafur, Gimeracil, Oteracil potassium (TS-1 $) 80 \mathrm{mg} /$ body. However, he suddenly complained of visual disturbance in his right eye about 8 months later (Fig. 3: Clinical course).

To evaluate the cause of the visual disturbance, fundoscopic examination and complete ophthalmologic examinations were performed (Fig. 4). Fundoscopic examination revealed profuse exudate, subretinal hemorrhage, and retinal detachment suggesting metastatic choroidal tumor. Ultrasonography showed retinal detachment by choroidal tumor, and optical coherence tomographic examination showed macular edema and irregular elevation of choroidal tissue. Magnetic resonance imaging (MRI) was also performed and a smooth and sharply marginated tumor was detected in his right ocular fundus in T1- and T2weighted images (Fig. 5). We could not detect any other origins for metastatic tumor of the choroid, although we performed CT, endoscopy, and FDG-PET.

We started the second line chemotherapy with weekly intravenous paclitaxel $(100 \mathrm{mg} / \mathrm{body})$ and intensitymodulated radiotherapy (IMRT) (40 Gy) to the right orbit. Although his retinal detachment was gradually worsened, sub-Tenon injection with dexamethasone was performed. Consequently, the retinal detachment was gradually improved and he was spared from going blind. There was a gradual decrease in carcinoembryonic antigen (CEA) from $7.9 \mathrm{ng} / \mathrm{ml}$ at diagnosis to $5.1 \mathrm{ng} / \mathrm{ml}$ after the chemoradiation therapy. He was continued to be treated with paclitaxel (PTX), 14 courses in total. At this point, we identified a complete response on PET and fundoscopic examination, and then we changed his chemotherapy to capecitabine ( $1200 \mathrm{mg} /$ body) alone. His recurrence-free survival period is 3 years until now (Fig. 3).

\section{Discussion}

It is considered that a number of reports about metastatic choroidal tumor have been increasing with the increase of cancer patients and improvement of survival rates with the advance of treatment. There are few reports about the cases from gastric adenocarcinoma [2-4]. Moreover, these reports just only show the choroidal metastases as one of the sites of systemic metastases, and there is no report about a solitary choroidal metastasis pointed out during adjuvant chemotherapy and achieved good local control.

Choroidal metastasis is considered to occur by hematogenous spread. This seems to be common in cases of lung cancer and breast cancer. In the English language literature, we could not find any previous articles about the risk or morbidity rate limited to only gastric cancer owing to its rarity. In our case, the microscopic vascular invasion of poorly differentiated adenocarcinoma or signet ring cell carcinoma was thought to be involved 

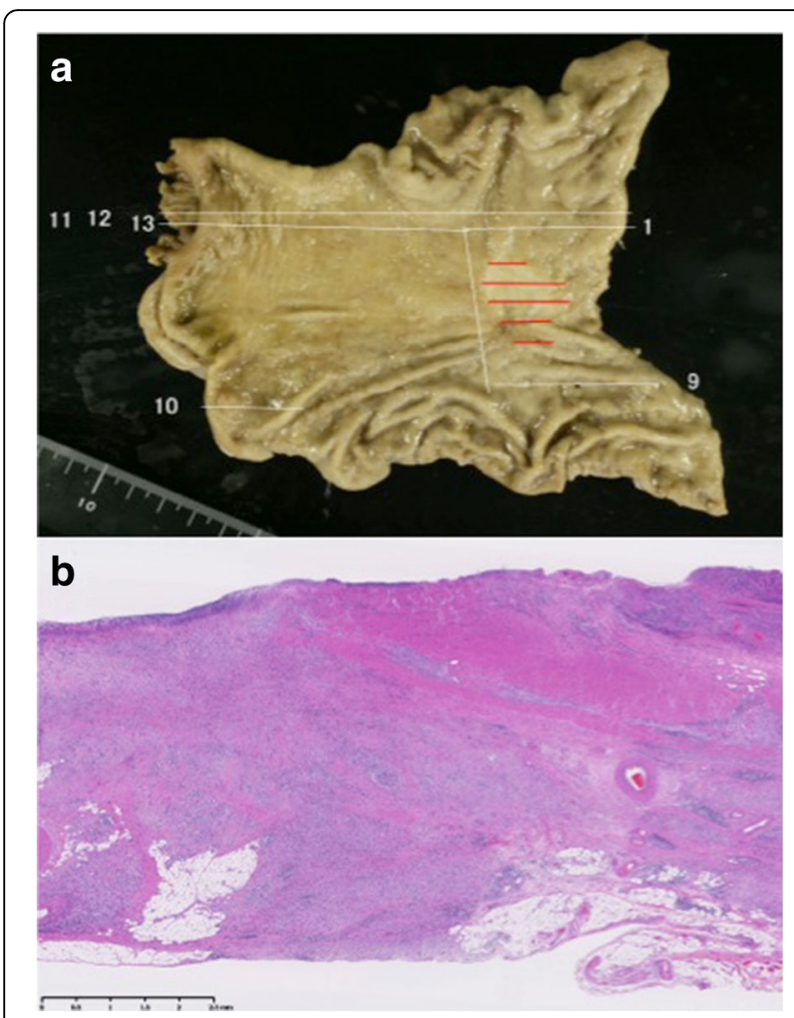

Fig. 2 Pathological findings. a Resected specimen shows a cancer measuring $30 \times 22 \mathrm{~mm}$ in the form of superficial depressed and excavated type. $\mathbf{b}$ Histopathological findings show poorly differentiated adenocarcinoma being exposed at the serosal surface only in the slice \#5 only (hematoxylin and eosin stain, $\times 40$ ) in the choroidal metastasis. Also, in approximately half of these patients, their primary sites are undetected despite the systematic evaluation, and they die of diffuse metastatic disease and their primary sites remain unknown [1].

In the diagnosis, it is important to differentiate between malignant melanoma and metastatic tumor, because melanoma and metastatic tumors are the two most common malignant choroidal tumors [5]. Malignant melanoma is seen as a localized lesion on fundoscopic examination in general; therefore, our findings were compatible with metastatic adenocarcinoma. In the literature, it has been reported that $\mathrm{N}$-isopropyl-p$\left({ }^{123} \mathrm{I}\right)$ iodoamphetamine single-photon emission computed tomography $\left({ }^{123}\right.$ I-IMP SPECT) and MRI are useful in distinguishing the two [6,7]. We were appreciative of the accuracy of MRI, and the T1-weighted image was isointense and the T2-weighted image was hypointense, so their findings were compatible with metastatic tumor, and not melanoma. Although we could not provide a definitive diagnosis to our case because of the difficulty of choroidal biopsy, we diagnosed metastasis of gastric adenocarcinoma with the findings from various imaging studies. Because some authors have reported the diagnosis by fundoscopic findings alone in previous literatures $[2,4]$, we assume that direct biopsy may not be necessary for diagnosing clinically.

Chemotherapy and external beam radiotherapy may be effective. In our case, chemoradiation therapy (CRT) achieved complete remission and saved the patient from going blind as well. It is reported that choroidal metastasis from gastric adenocarcinoma is almost resistant to various therapies and is presented with multiple organ

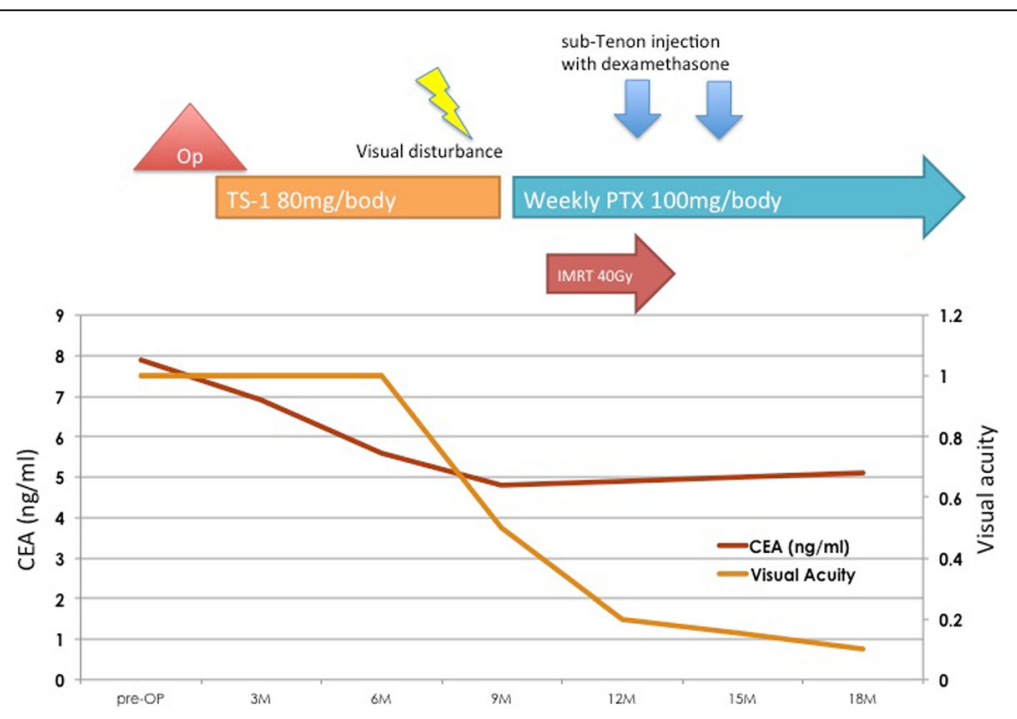

Fig. 3 Clinical course. (M: months; Op: operation; TS-1: Tegafur, Gimeracil, Oteracil potassium; PTX: paclitaxel; IMRT: intensity-modulated radiotherapy; CEA: carcinoembryonic antigen) 


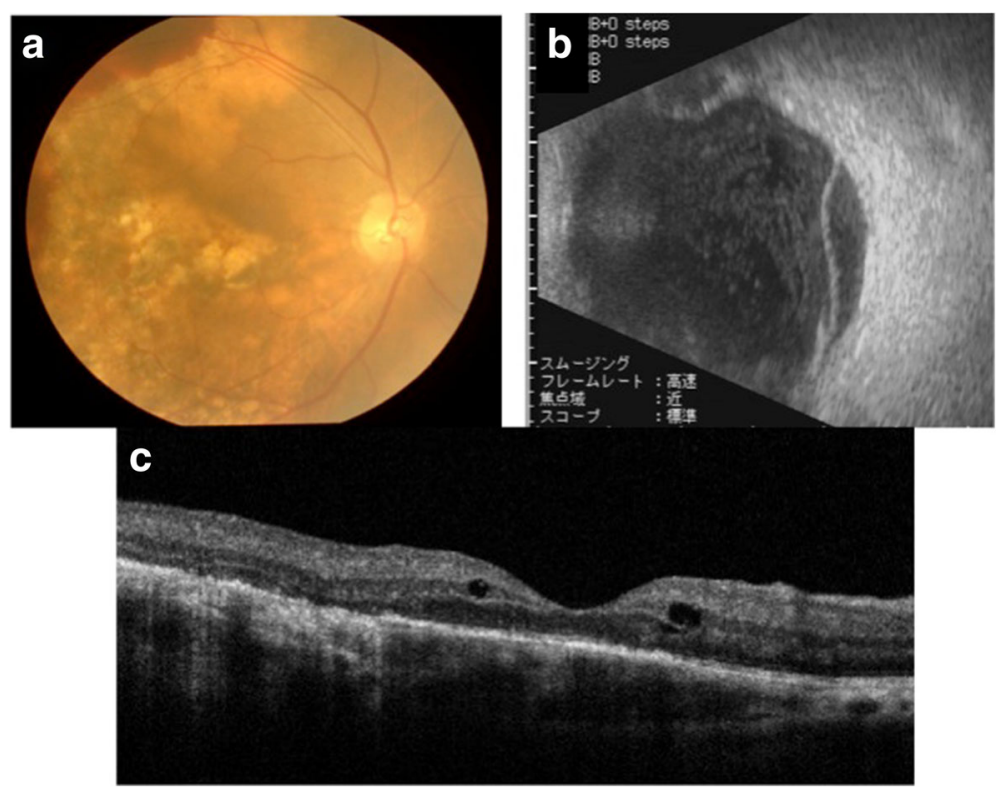

Fig. 4 Ophthalmologic examinations. a Fundoscopic examination. b Ultrasonography. c Optical coherence tomographic examination

metastasis and severe prognosis [1, 3]. It is interesting that multimodal therapy was effective in our case and local control for the metastatic ocular lesion was achieved. Although the issue here is how we can explain the success in the treatment in this case, it is reasonable to assume that the metastatic choroidal lesion was
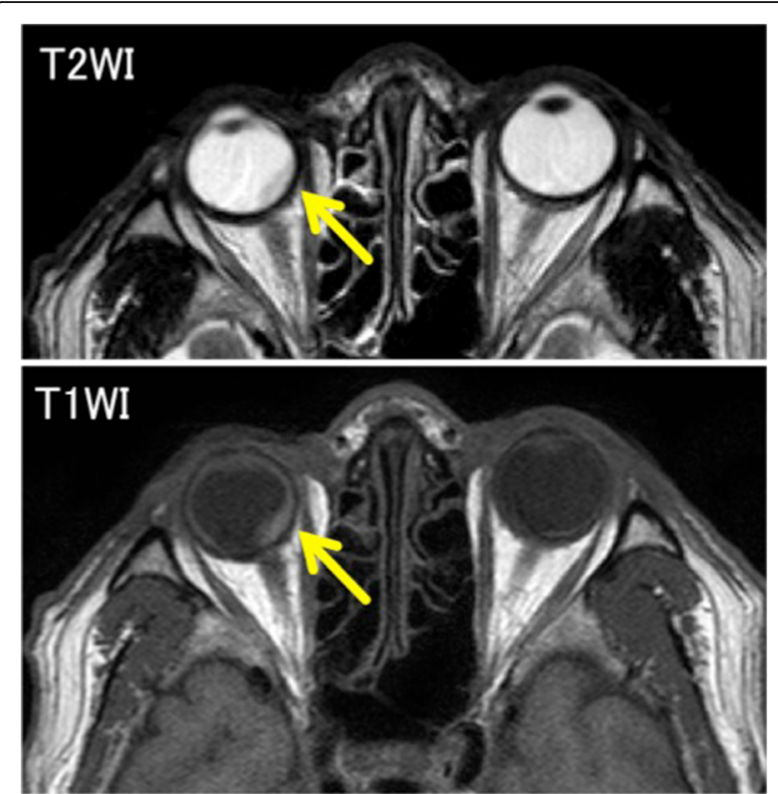

Fig. 5 Magnetic resonance imaging (MRI). An elevated choroidal tumor was detected. The T1-weighted image was isointense, and the T2-weighted image was hypointense fortunately localized within a small range and the symptom was detected in an early stage. The reason why we chose PTX is that we considered PTX as the second line. We thought standard TS-1 and cisplatin regimen will not be effective, because the choroidal metastasis was occurred during the adjuvant therapy with TS-1. Furthermore, we could not treat aggressively with a platinum-based combination regimen because of his chronic pancytopenia. On the other hand, we estimated that there is the risk of recurrence or other metastasis in several years, even once he had a complete remission. Although we debated whether or not to resume to treat with TS-1, TS-1 was predicted to have no effect in the first adjuvant chemotherapy. So, another oral anticancer drug that is approved for the management of gastric cancer was considered to be the best choice. Although there were few clinical trials for the efficacy of capecitabine for gastric cancer alone $[8,9]$, we considered capecitabine feasible for maintenance therapy after CRT. Currently, we intend to continue some kind of systematic chemotherapy for some time in the future with careful observation.

\section{Conclusions}

We reported a rare case of gastric adenocarcinoma who presented with unilateral solitary choroidal metastasis during adjuvant chemotherapy. Therefore, it is important to be aware of the possibility of a metastatic choroidal tumor, when visual disturbances do arise during gastric cancer treatment. 


\begin{abstract}
Abbreviations
${ }^{123}$ I-IMP SPECT: N-isopropyl-p- $\left({ }^{123} \mid\right)$ iodoamphetamine single-photon emission computed tomography; CEA: Carcinoembryonic antigen; CRT: Chemoradiation therapy; CT: Computed tomography; DM: Distal margin; FDG-PET: Fluorodeoxyglucose-positron emission tomography; HER2: Human epidermal growth factor receptor 2; IMRT: Intensitymodulated radiotherapy; INF: Pattern of infiltrating growth; MRI: Magnetic resonance imaging; PM: Proximal margin; por2: Non solid type poorly differentiated adenocarcinoma; PTX: Paclitaxel; sci: Scirrhous type; sig: Signet-ring cell carcinoma; T1b: Tumor invades the submucosal layer; T4a: Tumor exposes on the surface of serosa; TS-1 $1^{\circ}$ : Tegafur, Gimeracil, Oteracil potassium
\end{abstract}

\title{
Authors' contributions
}

MS contributed to this work and wrote the paper. MS, SM, TT, and YK treated the patient and collected the patient's clinical data. HS, YF, YM, and TM supported this work and provided advice. All authors read and approved the final manuscript.

\section{Consent for publication}

Written informed consent was obtained for this publication and accompanying images from the patient involved.

\section{Competing interests}

The authors declare that they have no competing interests.

\section{Author details}

'Department of Surgery, Fukui-ken Saiseikai Hospital, 7-1

Wadanaka-Funabashi, Fukui 918-8503, Japan. ${ }^{2}$ Department of Ophthalmology, Fukui-ken Saiseikai Hospital, Fukui 918-8503, Japan.

${ }^{3}$ Department of Radiation therapy, Fukui-ken Saiseikai Hospital, Fukui

918-8503, Japan

Received: 29 December 2016 Accepted: 18 February 2017

Published online: 21 February 2017

\section{References}

1. Shields CL, Shields JA, Gross NE, Schwartz GP, Lally SE. Survey of 520 eyes with choroidal metastases. Ophthalmology. 1997;104:1265-76.

2. Sahin A, Kiratli $\mathrm{H}$. Choroidal metastasis as a first sign of recurrence in patient with gastric adenocarcinoma. Can J Ophthalmol. 2007:42:331-2.

3. Melo DH, Pierre Filho Pde T, Gomes PR, Holanda AG, Amadei LP. Choroida metastasis of gastric adenocarcinoma as a first sign of systematic disease recurrence: case report. Arq Bras Oftalmol. 2010;73:467-8.

4. Kawai S, Nishida T, Hayashi Y, Ezaki H, Yamada T, et al. Choroidal and cutaneous metastasis from gastric adenocarcinoma. World J Gastroenterol. 2013;19:1485-8.

5. Tong KA, Osborn AG, Mamalis N, Harrie RP, Call NB. Ocular melanoma. AJNR Am J Neuroradiol. 1993:14:1359-66.

6. Goto H. Clinical efficacy of 1231-IMP SPECT for the diagnosis of malignant uveal melanoma. Int J Clin Oncol. 2004;9:74-8.

7. Peyster RG, Augsburger JJ, Shields JA, Hershey BL, Eagle R, Haskin ME. Intraocular tumors: evaluation with MR imaging. Radiology. 1988;168:773-9.

8. Koizumi W, Saigenji K, Ujiie S, Terashima M, Sakata Y, et al. A pilot phase II study of capecitabine in advanced or recurrent gastric cancer. Oncology. 2003:64:232-6

9. Lee JL, Kang YK, Kang HJ, Lee KH, Zang DY, Ryoo BY, et al. A randomised multicentre phase II trial of capecitabine vs S-1 as first line treatment in elderly patients with metastatic or recurrent unresectable gastric cancer. Brt J Cancer. 2008;99:584-90.

\section{Submit your manuscript to a SpringerOpen ${ }^{\circ}$ journal and benefit from:}

- Convenient online submission

- Rigorous peer review

- Immediate publication on acceptance

- Open access: articles freely available online

- High visibility within the field

- Retaining the copyright to your article 